OPEN ACCESS

Edited by:

Weiliang Wang,

Sun Yat-sen University, China

Reviewed by:

Zhongming Wei,

Institute of Semiconductors (CAS),

China

Zexiang Deng,

The University of Hong Kong, Hong

Kong, SAR China

Haiming Huang,

Guangzhou University, China

Mehmet Yagmurcukardes,

University of Antwerp, Belgium

*Correspondence:

Chun-Gang Duan

cgduan@clpm.ecnu.edu.cn

Specialty section:

This article was submitted to

Thin Solid Films,

a section of the journal

Frontiers in Materials

Received: 04 July 2021

Accepted: 25 August 2021

Published: 10 September 2021

Citation:

Zhao Y-F, Guan Z, Zhong N, Yue F-Y, Xiang P-H and Duan C-G (2021)

Raman Spectra of Bulk and Few-Layer GeSe From First-

Principles Calculations.

Front. Mater. 8:736057.

doi: 10.3389/fmats.2021.736057

\section{Raman Spectra of Bulk and Few-Layer GeSe From First-Principles Calculations}

\author{
Yi-Feng Zhao ${ }^{1}$, Zhao Guan ${ }^{1}$, Ni Zhong ${ }^{1,2}$, Fang-Yu Yue ${ }^{1}$, Ping-Hua Xiang ${ }^{1}$ and \\ Chun-Gang Duan ${ }^{1,2 *}$
}

${ }^{1}$ Key Laboratory of Polar Materials and Devices (MOE) and Department of Electronics, East China Normal University, Shanghai, China, ${ }^{2}$ Collaborative Innovation Center of Extreme Optics, Shanxi University, Taiyuan, China

Raman spectra play a significant role in the study of polar materials. Herein, we report the influence of strain and interlayer shift on vibration responses in bulk and few-layer ferrovalley material GeSe in different polarization states (ferroelectric/FE and antiferroelectric/AFE) based on density functional theory and density functional perturbation theory calculations. We find $A_{g}{ }^{1}$ mode shifts by about $10 \mathrm{~cm}^{-1}$ from monolayer to bilayer and trilayer due to the interlayer coupling. The $A_{g}{ }^{3}$ mode on behalf of FE mode is observed that is consistent with the experiments in bulk and fewlayer GeSe. Meanwhile, in our calculations, with the transition between AFE and FE state in the bilayer and trilayer, the Raman frequency of $A_{g}{ }^{2}$ and $A_{g}{ }^{3}$ mode decrease obviously whereas that of $A_{g}{ }^{1}$ mode increases. Interestingly, the Raman peaks shifted a lot due to the strain effect. We expect these variations in the Raman spectroscopy can be employed to identify the status of GeSe films, e.g., the AFE or FE state, and the number of layers in experiments.

Keywords: GESE, Raman spectra, first-principales calculation, ferroelectric, antiferroelectric, ferrovalley

\section{INTRODUCTION}

Ferroelectric (FE) materials with a stable spontaneous polarization that could be switched under external electric field have been widely studied and exploited in multifunctional devices such as ferroelectric synapse, field-effect transistors, and ferroelectric tunnel junction (Scott and Paz de Araujo, 1989; Mathews et al., 1997; Velev et al., 2007; Garcia et al., 2009; Huang et al., 2018b; Shen et al., 2019; Tian et al., 2019; Guan et al., 2020) due to their abundant physics. Until now, perovskite ferroelectric oxides are mostly utilized in these devices. However, with the decrease of thickness, ferroelectricity can hardly be maintained because of the existence of depolarization field in ferroelectric films (Junquera and Ghosez, 2003), which severely restrain the application of traditional perovskite ferroelectrics in miniaturized and high-density devices. In addition, the interfacial defect caused by lattice mismatch can also destroy the film ferroelectricity (Duan et al., 2006; Wang et al., 2010). The emergence of twodimensional (2D) ferroelectrics provides an opportunity to resolve these difficulties (Wu and Jena, 2018). Recently, plenty of 2D ferroelectrics have been successfully exfoliated from a bulk structure in experiments, including in-plane ferroelectricity (Chang et al., 2016; Chang et al., 2019; Higashitarumizu et al., 2020), and out-of-plane ferroelectricity (Liu et al., 2016; Zhou et al., 2017; You et al., 2019; Yuan et al., 2019). Beyond experimental works, theoretical studies have predicted that ferroelectricity can survive in 2D materials (Ding et al., 2017; Huang et al., 
2018a), in which some even possess noncollinear ferroelectric ordering (Lin et al., 2019; Song et al., 2021).

Among these works, group IV chalcogenides ( $M X, M=S n, G e ;$ $\mathrm{X}=\mathrm{S}, \mathrm{Se}$ ) with few-layer have been discovered with intrinsic ferroelectricity and antiferroelectricity in the experiment (Fei et al., 2016), with a fantastic optical selective property as a polarizer (Shen et al., 2018), valley physics (Rodin et al., 2016), a high absorption coefficient as photovoltaic cells (Franzman et al., 2010; Shi and Kioupakis, 2015), robust ferroelectricity as nonvolatile storage (Wang and Qian, 2017) and so on (Yagmurcukardes et al., 2016). On the other hand, FE and antiferroelectric (AFE) phase transition is also predicted by interlayer sliding (Xu et al., 2021), suggesting an AFE tunnel junction can be realized in these materials (Ding et al., 2021).

As a most general and powerful tool to study crystal structures and vibration properties, Raman spectroscopy has been diffusely employed due to the advantage of nondestructive to the sample and easy sample preparation. Especially in 2D materials, the Raman spectrum can be used to precisely identify the number of layers because of its high sensitivity of thickness. For instance, in graphene (Ferrari et al., 2006), the $G$ peak down-shifts with the decrease of layers, which is a sign to determine the number of layers. A similar case occurs in the theoretical work of $\mathrm{PbI}_{2}$ (Yagmurcukardes et al., 2018), the larger number of layers, the more blueshift with the Raman peak of $A_{g}{ }^{1}$ mode. On the contrary, a redshift of $A_{g}$ and $B_{1 g}$ modes with the increased layers in few-layer phosphorene (Feng et al., 2015) could be observed. Moreover, the information of interlayer stacking and an effect of external field can be also detected by Raman spectroscopy (Zhang et al., 2015). Therefore, investigation using Raman spectroscopy could help to understand charming properties in 2D materials (Deng et al., 2019; Saboori et al., 2019).

However, it should be mentioned that few-layer GeSe is rarely explored in the experiment due to the great challenge for sample preparation, leading to difficulty to study their layer numbers and phase transition related Raman spectroscopy. Consequently, systematic study of Raman spectroscopy of layered GeSe is necessary by theoretical calculation, which can provide guidelines for evaluating phonon-related characterization of GeSe based on Raman spectroscopy.

In this paper, we fully investigate the Raman spectroscopy, concerning the bulk and few-layer GeSe from monolayer to trilayer, by first-principles calculations. The van der Waals (vdW) correction is adopted in our calculations by comparing the Raman spectrum and crystal structure with experiment results in bulk GeSe. We find four vibration modes of $A_{g}{ }^{1}, B_{3 g}$, $A_{g}{ }^{2}$ and $A_{g}{ }^{3}$ in bulk GeSe, in which $A_{g}{ }^{2}$ is absent in the experiment but exists in SnSe (Yang et al., 2018). For monolayer GeSe, these vibration peaks are located at $81.84 \mathrm{~cm}^{-1}, 97.54 \mathrm{~cm}^{-1}$, $141.94 \mathrm{~cm}^{-1}$ and $183.14 \mathrm{~cm}^{-1}$, respectively. With the increase of layer number, $A_{g}{ }^{1}$ mode has a redshift but $A_{g}{ }^{2}$ and $A_{g}{ }^{3}$ exhibit a large blue shift as large as $40 \mathrm{~cm}^{-1}$ and $30 \mathrm{~cm}^{-1}$, which verify the importance of interlayer interaction and can be used to identify the thickness of GeSe. In addition, we find strain has a remarkable influence on the Raman spectrum in different structures, suggestive of the possibility to probe the strain effect using optical method. More importantly, AFE/FE phase transition could be triggered by some methods such as interlayer sliding or strain. Herein, we use interlayer shift to realize the transition in few-layer as well as study the Raman spectroscopy on transition structures. We find an $A_{g}{ }^{1}$ mode increase of about $10 \mathrm{~cm}^{-1}$ whereas $A_{g}{ }^{2}$ and $A_{g}{ }^{3}$ decrease with the transition from AFE to FE phase, helping us to determine the AFE state or FE state. Our calculation could give information about the number of layers, whether the system under strain state and the ferroelectric phase of layered GeSe.

\section{CALCULATION DETAILS}

The optimized structures are calculated by employing the PWSCF package of the QUANTUM-ESPRESSO (Giannozzi et al., 2009) within the density functional theory (DFT) (Hohenberg and Kohn, 1964; Kohn and Sham, 1965). We adopt the local density approximation (LDA) with Perdew-Zunger parametrization (Perdew and Zunger, 1981) and the generalized gradient approximation (GGA) with PerdewBurke-Ernzehof parametrization (Perdew et al., 1996) to evaluate the exchange-correlation energy and consider a modified norm-conserving pseudopotential to describe the valence electron-ion interactions (Gonze et al., 1991). In our calculations, the vdW correction was fully taken into account by using the DFT + D2 method (Grimme, 2006; Barone et al., 2009). To avoid the spurious interactions between periodic images, vacuum spacing of $20 \AA$ was set along the c-direction. The energy cutoff was set to $50 \mathrm{Ry}$ and a Brillouin zone (BZ) integration is adopted with a k-grid density of $7 \times 7 \times 3$ for bulk structures and $7 \times 7 \times 1$ for few-layer structures via using the k-points scheme. All structures are optimized until the HellmanFeynman force is below $10^{-6} \mathrm{Ry} / \mathrm{Bohr}$ and the convergence of electric energy is of about $10^{-4}$ Ry/atom.

The related phonon vibration frequencies are calculated by diagonalizing the force constant matrix within the density functional perturbation theory (DFPT) (Baroni et al., 2001). The BZ integration is adopted with a k-grid density of $14 \times 14 \times 6$ for bulk structures and $14 \times 14 \times 1$ for few-layer structures at gamma point. The force tolerance is set to $10^{-10}$.

The Raman intensity of Raman activate mode (Supplementary Figures S1,S2) can be written as (Umari et al., 2001; Ceriotti et al., 2006):

$$
I \propto\left|e_{s} \cdot R \cdot e_{i}\right|^{2}
$$

where $e_{\mathrm{s}}$ and $e_{\mathrm{i}}$ represent the electric polarization vectors of incident and scattered light, respectively.

\section{RESULTS}

\section{Raman Active Modes of Bulk and Few-Layer GeSe}

Bulk GeSe belongs to a layered structure in $\mathrm{AB}$ stacking with vdW interactions, as plotted in Figure 1A, with the space group of Pnma. We noted the longer axis in the $x-y$ plane is the armchair 

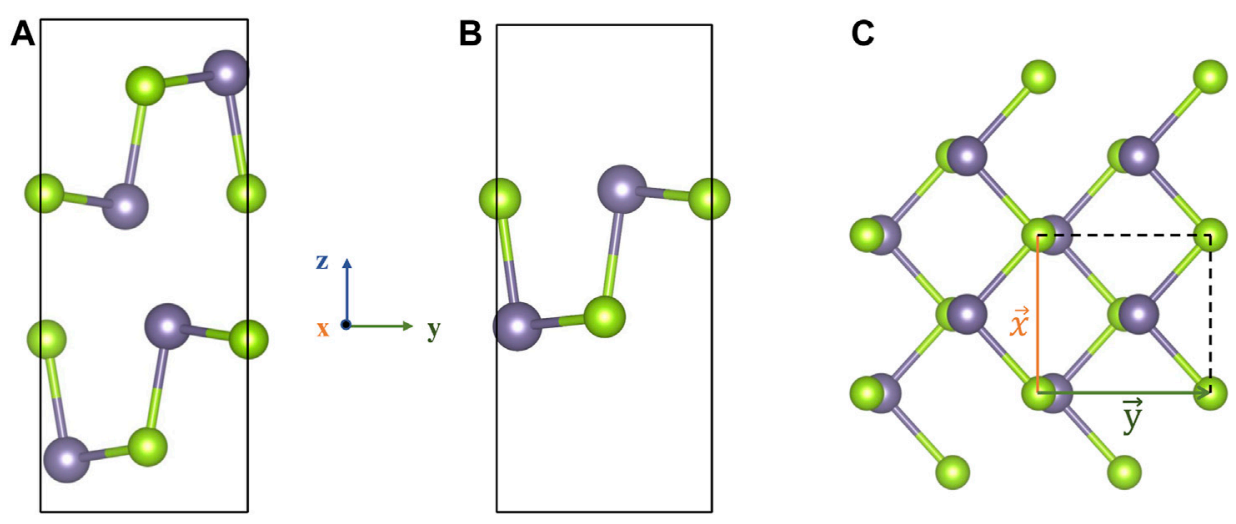

FIGURE 1 | The atomic structures of (A) bulk and (B) monolayer GeSe. (C) The top view of monolayer GeSe. The dark and green spheres represent Ge and Se atoms, respectively.

TABLE 1 | The calculated structure lattice parameters (in the unit of $\AA$ ) of bulk GeSe with various methods.

\begin{tabular}{|c|c|c|c|c|}
\hline Bulk & Method & $a$ & $b$ & $c$ \\
\hline & LDA & 3.815 & 4.215 & 10.597 \\
\hline & GGA & 3.899 & 4.278 & 11.169 \\
\hline & vdW-D2 & 3.845 & 4.481 & 11.024 \\
\hline & vdW-D3 & 3.888 & 4.531 & 11.246 \\
\hline & Exp & 3.833 & 4.388 & 10.825 \\
\hline
\end{tabular}

direction $(y)$ and the other axis is the zigzag $(x)$ direction. Figures 1B,C shows the monolayer GeSe that retains the symmetry of the bulk structure. The relative displacement between the Ge atom and Se atom indicates the system is in the ferroelectric phase.

We first optimize the lattice parameters of the bulk structure to compare to experimental lattices, shown in Table1. As we can see, there is a large lattice difference of the $b$ axis (b axis is the armchair direction) under various pseudopotentials and methods for bulk structure. DFT-D2 (vdW-D2) and DFT-D3 (vdW-D3) method of Grimme is applied as vdW correction (Grimme, 2006). The optimized structure with the vdW-D2 method is in good agreement with experimental results compared to others. As to LDA and GGA methods, the lattice parameters is underestimated by 4 and $2.5 \%$ of the b axis, respectively. Based on these calculated structures, we investigate the Raman frequencies, and we find vdW-D2 gives a credible result in comparison to other methods, consistent with previous work (Park et al., 2019). Hence the interlayer interactions will be considered in the following calculations of bulk and multilayer structures. We should note that the $A_{g}{ }^{2}$ mode obtained in theoretical calculations was not observed in experiments on GeSe (Wang et al., 2017) but appears in the similar system SnSe (Yang et al., 2018), which is due to the Raman tensor of $A_{g}{ }^{2}$ mode is too small for its Raman peak to be observed in GeSe.

According to the above comparison and analysis, we use the vdW-D2 method to relax our structure and calculate the optical phonon frequencies. To understand the Raman frequencies of
TABLE 2 | The Raman vibration frequency (in the unit of $\mathrm{cm}^{-1}$ ) of bulk GeSe with different methods.

\begin{tabular}{|c|c|c|c|c|c|}
\hline Bulk & Method & $A_{g}{ }^{1}$ & $B_{3 g}$ & $A_{g}{ }^{2}$ & $A_{g}{ }^{3}$ \\
\hline & LDA & 70.83 & 145.19 & 176.96 & 185.53 \\
\hline & GGA & 67.6 & 141.02 & 164.76 & 182.25 \\
\hline & vdW-D2 & 68.21 & 153.02 & 179.05 & 189.6 \\
\hline & vdW-D3 & 67.80 & 143.44 & 163.84 & 184.23 \\
\hline & Exp & 80 & 150 & & 188 \\
\hline
\end{tabular}

Note: Previous experiment results of lattice parameters and Raman vibration frequency are listed for comparison (Wang et al., 2017).

bulk GeSe, we analyze the crystal structure by combining the irreducible representations of $\Gamma$ points. The primitive unit cell of bulk GeSe include eight atoms, resulting in 24 vibrational modes as following:

$$
\text { Tacoustic }=B_{1 u}+B_{2 u}+B_{3 u}
$$

and

$$
\text { Гoptic }=3 B_{1 u}+B_{2 u}+3 B_{3 u}+2 A_{u}+4 A_{g}+2 B_{1 g}+4 B_{2 g}+2 B_{3 g}
$$

which are three acoustical $\left(B_{1 u}, B_{2 u}, B_{3 u}\right)$, seven infrared active modes $\left(3 B_{1 u}, B_{2 u}, 3 B_{3 u}\right)$, two silent modes $\left(2 A_{u}\right)$, as well as twelve Raman modes $\left(4 A_{g}, 2 B_{1 g}, 4 B_{2 g}, 2 B_{3 g}\right)$. Four active Raman modes for bulk GeSe as $3 A_{g}$ at $68.21 \mathrm{~cm}^{-1}, 179.05 \mathrm{~cm}^{-1}$, and $189.6 \mathrm{~cm}^{-1}$ and $B_{3 g}$ at $153.02 \mathrm{~cm}^{-1}$ are listed in Table 2, and we define $3 A_{g}$ as $A_{g}{ }^{1}, A_{g}{ }^{2}$ and $A_{g}{ }^{3}$, respectively. To study the four active phonon vibration modes, we projected the eigenvectors of the dynamical matrix on $\mathrm{Ge}$ and $\mathrm{Se}$ atoms. Figure 2 gives the outline of the four vibration active modes of bulk GeSe. We find that $A_{g}{ }^{1}, A_{g}{ }^{2}$ and $A_{g}{ }^{3}$ couple the in-plane and out-of-plane vibrations with various contribution. $A_{g}{ }^{1}$ and $A_{g}{ }^{2}$ modes are mainly dominated by out-of-plane motion, in which $\mathrm{Ge}$ and Se atoms (Ge1 and Se2) move to the opposite direction of $A_{g}{ }^{1}$ mode whereas in $A_{g}{ }^{2}$ mode they move to the same direction. $A_{g}{ }^{3}$ mode is most contributed by armchair direction, and only the $\mathrm{Ge}$ atom has a small out-of-plane contribution. For $A_{g}{ }^{3}$ mode, it 

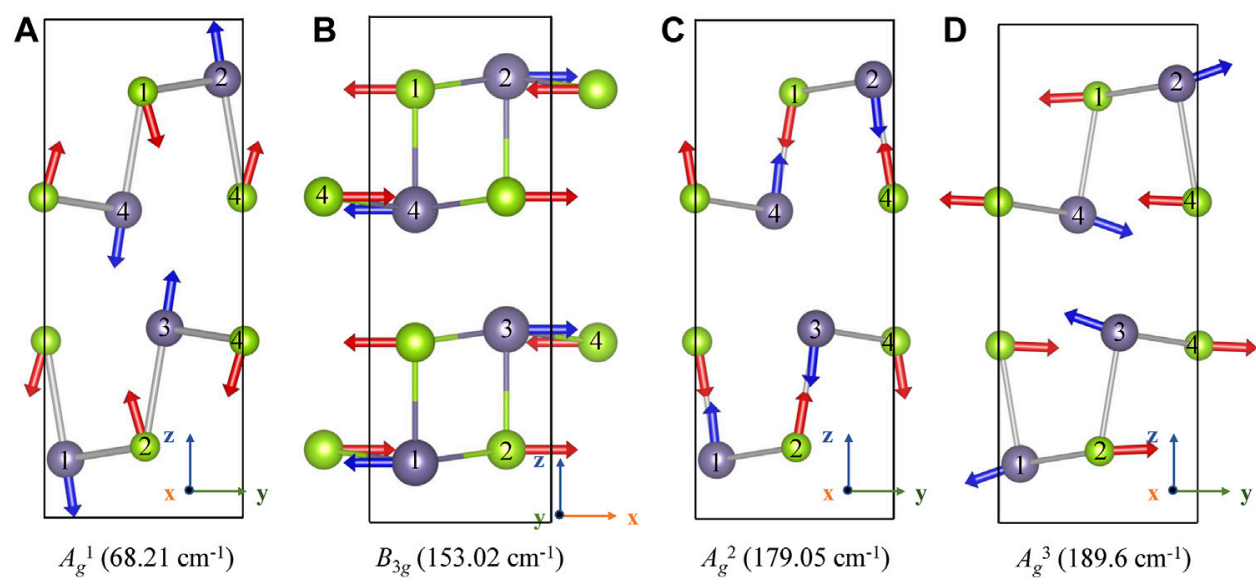

FIGURE 2 | The activate Raman vibration mode of bulk GeSe. The out-of-plane along z direction.

TABLE 3 | The lattice constant and activate Raman frequency (in the unit of $\AA$ and $\mathrm{cm}^{-1}$ ) for monolayer, bilayer and trilayer GeSe.

\begin{tabular}{lcccccc}
$\begin{array}{l}\text { Number of } \\
\text { layers }\end{array}$ & $\boldsymbol{a}$ & $\boldsymbol{b}$ & $\boldsymbol{A}_{\boldsymbol{g}}{ }^{\mathbf{1}}$ & $\mathbf{B}_{\boldsymbol{3 g}}$ & $\boldsymbol{A}_{\boldsymbol{g}}{ }^{2}$ & $\boldsymbol{A}_{\boldsymbol{g}}{ }^{\mathbf{3}}$ \\
\hline $\begin{array}{l}\text { monlayer } \\
\text { bilayer }\end{array}$ & 3.986 & 4.258 & 81.84 & 97.54 & 141.94 & 183.14 \\
trilayer & 3.873 & 4.428 & 69.26 & 140.77 & 176.59 & 185.29 \\
& 3.853 & 4.478 & 69.66 & 137.18 & 179.89 & 185.82 \\
\end{tabular}

forms by an in-plane vibration along the armchair direction, indicating an intrinsic ferroelectric vibration mode in one layer of bulk GeSe, which agrees with previous experiment work (Zhao et al., 2018).

Monolayer GeSe has been predicted a member of ferrovalley materials with plenty of fantastic physical properties (MoralesFerreiro et al., 2017; Shen et al., 2018; Liu et al., 2021). Thus, it is necessary to investigate the Raman spectrum dependence of fewlayer. For monolayer GeSe, the four active Raman peaks of $A_{g}{ }^{1}$, $B_{3 g}, A_{g}{ }^{2}$, and $A_{g}{ }^{3}$ modes are located at $81.84 \mathrm{~cm}^{-1}, 97.54 \mathrm{~cm}^{-1}$,
$141.94 \mathrm{~cm}^{-1}$, and $183.14 \mathrm{~cm}^{-1}$ illustrated in Table 3. Even though, both of paraelectric and ferroelectric phases exist in monolayer $\mathrm{GeSe}$, we investigate the FE phase in the current work due to its lower energy state and stable structure. With the increase of thickness from monolayer to trilayer, the Raman shift changes a lot. Indeed, $A_{g}{ }^{1}$ mode generates a redshift from $81.84 \mathrm{~cm}^{-1}$ in the monolayer to $69.26 \mathrm{~cm}^{-1}$ in the bilayer. As to the remanent modes, they all increase. However, few shifts are induced between bilayer and trilayer. Our calculation suggests that GeSe has a large Raman shift with increasing layer thickness compared to other 2D materials (Lee et al., 2010; Tan et al., 2012; Yagmurcukardes et al., 2018; Kong et al., 2021). It provides a valuable approach for distinguishing the structure between monolayer and few-layer GeSe. The reason for such a large discrepancy of Raman peaks between monolayer and multilayer is attributed to the interlayer vdW interactions in multilayer GeSe, giving rise to structures difference. As a result, the lattice parameters that is listed in Table 3 along the armchair direction of monolayer GeSe are largely smaller than that of bilayer and trilayer.
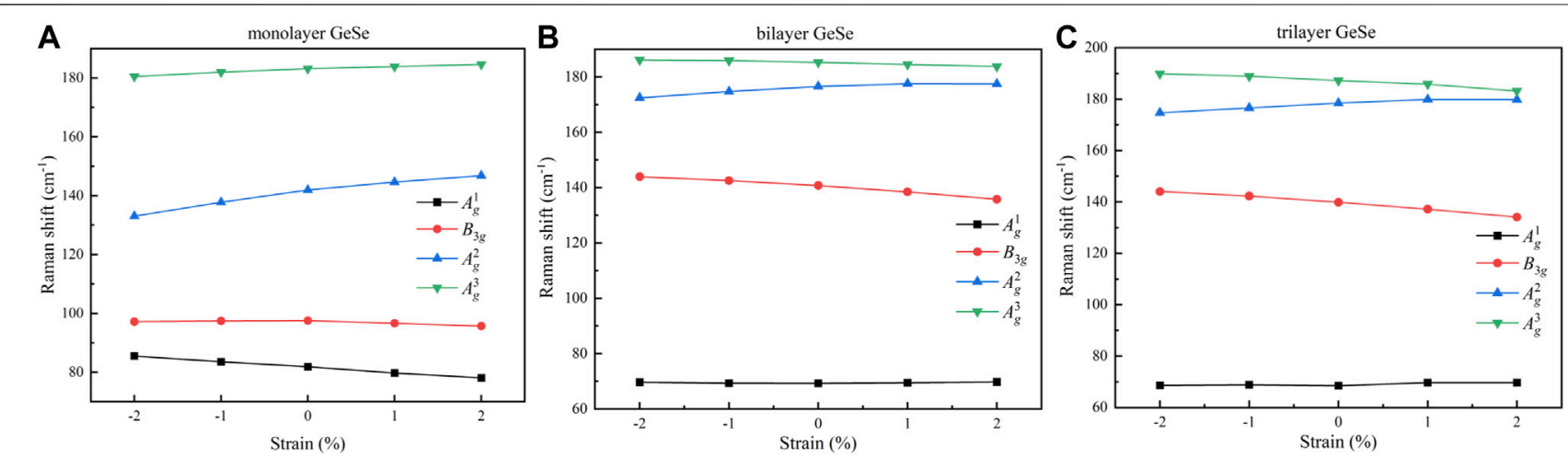

FIGURE 3 | The influence of the Raman frequency on strain from -2 to $2 \%$ for (A) monolayer, (B) bilayer and (C) trilayer GeSe. 

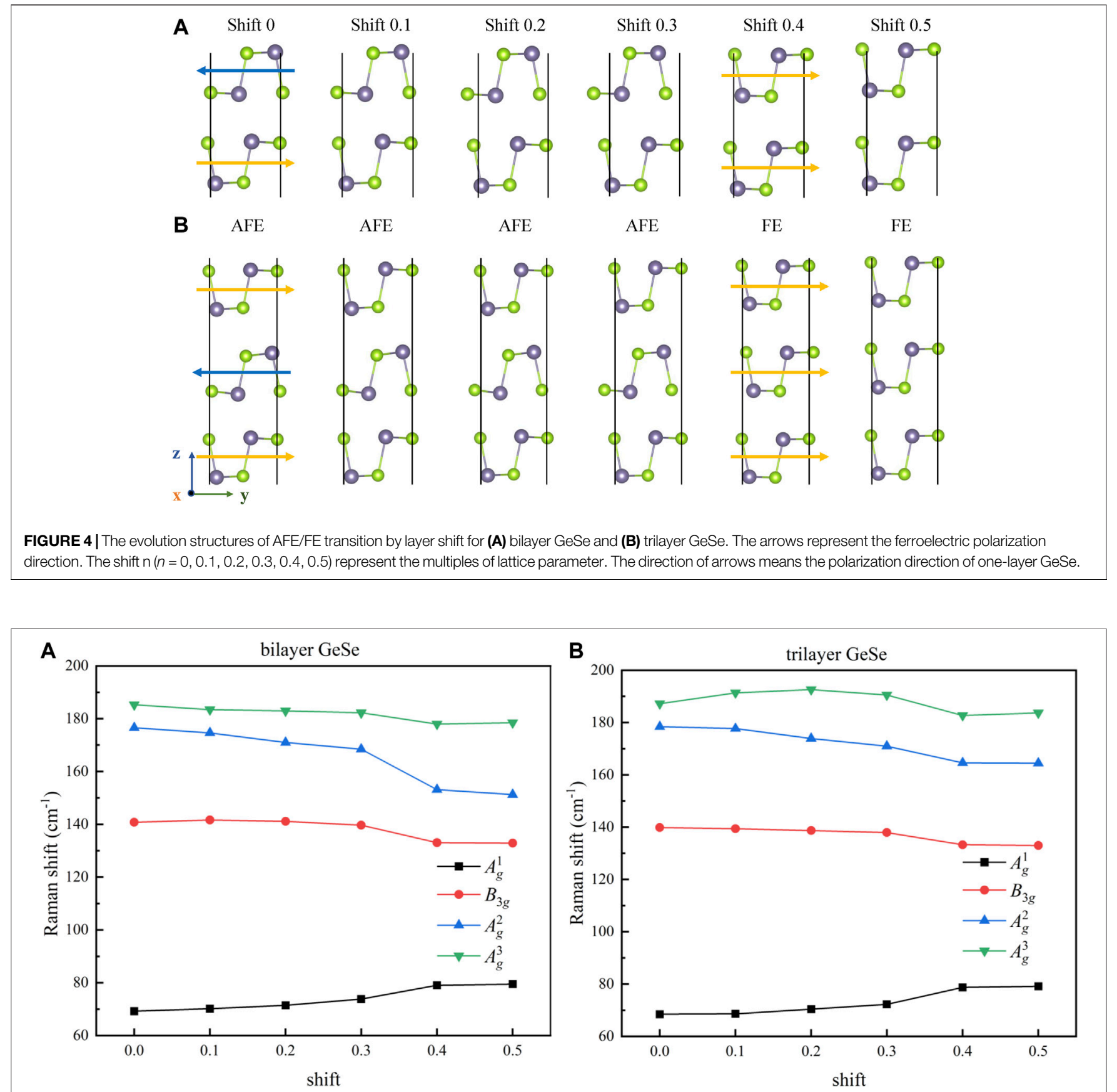

FIGURE 5 | The activate Raman frequency related to the evolution structures for (A) bilayer and (B) trilayer GeSe in Figure 4.

\section{The Influence of Strain on Raman Peaks}

Considering materials always are in strain in heterostructure or different temperatures, the influence of strain on Raman active modes is further explored in bulk and few-layer GeSe by changing the lattice parameter on both armchair and zigzag direction, as depicted in Figure 3. In monolayer GeSe, with the varying strain from -2 to $2 \%$, the vibration mode of $A_{g}{ }^{1}$ linearly decreases in frequency. On the contrary, $A_{g}{ }^{2}$ and $A_{g}{ }^{3}$ modes exhibit a clear increase with strain. And for $B_{3 g}$ mode, an inconspicuous change has been observed. Raman peaks between bilayer and trilayer GeSe possess the same trend in tensile and compressive strains. It should be mentioned that Raman frequency of $A_{g}{ }^{1}$ mode is most steady and rarely changed compared with the other three modes. Whereas, $B_{3 g}$ and $A_{g}{ }^{3}$ modes increase by compressive strain and decrease along with tensile strain in Figures 3B,C. Moreover, as the strain increases, 
the gap of Raman shift among $A_{g}{ }^{2}$ and $A_{g}{ }^{3}$ modes is shrunk. Our results suggest that the stain has a great effect on the Raman shift, which can be detected in the experiment, demonstrating the stress dependence of Raman spectra can also be used for the determination of crystal direction.

\section{Interlayer Shift-Induced Raman Shift}

Besides FE phase, AFE phase GeSe is also considered to be the lowest energy phase in an even number of layers. However, in some ways, the FE phase is more desired by researchers to utilize in multifunctional devices. To date, only the theoretical studies of AFE/FE transition for GeSe multilayers have been reported rather than any experimental works (Xu et al., 2021). Therefore, it is remarkable things to investigate the interlayer shift-induced AFE/FE transition and relevant Raman shift in few-layer GeSe. To realize the phase transition, we consider the AFE phase as a start structure. The structure evolution of the AFE/FE transition is illustrated in Figure 4. In bilayer GeSe, the bottom layer GeSe is fixed and the top layer artificially shifted along- $y$-direction in Figure 4A. When the top layer was shifted about $0.4 \mathrm{~b}$ (b is the lattice parameter of $y$-direction), the system translates to an FE state. The same phenomenon appears in trilayer GeSe, in which we move interlayer between the top and bottom layer that is in fixation, showing in Figure 4B. Each shifted structure has been fully relaxed so that the Raman spectrum is obtained with a stable structure. Figures 5A,B represents the Raman shift in the intermediate phase. $A_{g}{ }^{1}$ mode of FE phase increases by of about $10 \mathrm{~cm}^{-1}$ compared with AFE phase in both bilayer and trilayer GeSe. Meanwhile. $B_{3 g}, A_{g}^{2}$, and $A_{g}^{3}$ mode show a redshift, in which the same variation rule in $A_{g}^{2}$ mode is observed in both of bilayer and trilayer $\mathrm{GeSe}$. In bilayer GeSe, $A_{g}{ }^{3}$ mode decreases with a nearly linear slope, yet it firstly increases and then diminishes with the interlayer shift of the trilayer. These large discrepancies in the Raman shift could be taken as an explicit evidence to identify the induced AFE/FE transition in the experiment.

\section{CONCLUSION}

To summarize, the Raman frequency of few-layer and bulk GeSe is systematically investigated using first-principles calculations. A large difference of Raman peaks between the monolayer and the

\section{REFERENCES}

Barone, V., Casarin, M., Forrer, D., Pavone, M., Sambi, M., and Vittadini, A. (2009). Role and Effective Treatment of Dispersive Forces in Materials: Polyethylene and Graphite Crystals as Test Cases. J. Comput. Chem. 30 (6), 934-939. doi:10.1002/jcc.21112

Baroni, S., de Gironcoli, S., Dal Corso, A., and Giannozzi, P. (2001). Phonons and Related crystal Properties from Density-Functional Perturbation Theory. Rev. Mod. Phys. 73 (2), 515-562. doi:10.1103/RevModPhys.73.515

Ceriotti, M., Pietrucci, F., and Bernasconi, M. (2006). Ab Initiostudy of the Vibrational Properties of crystallineTeO2: Thea, $\beta$, And $\gamma$ phases. Phys. Rev. B 73 (10), 104304. doi:10.1103/PhysRevB.73.104304

Chang, K., Liu, J., Lin, H., Wang, N., Zhao, K., Zhang, A., et al. (2016). Discovery of Robust In-Plane Ferroelectricity in Atomic-Thick SnTe. Science 353 (6296), 274-278. doi:10.1126/science.aad8609 multilayer, demonstrating the significant effect of vdW interlayer interaction. We then find the strain influence on these systems, in which $A_{g}{ }^{2}$ linearly increases and $B_{3 g}$ mode linearly diminishes from -2 to $2 \%$. More important, the interlayer shift could also induce a phase transition from AFE to FE. As a result, the Raman frequency discrepancy of four vibration modes is natural. We truly expect our calculations can pave a way to verify the number of layers, strain influence, and polarity in few-layer GeSe.

\section{DATA AVAILABILITY STATEMENT}

The raw data supporting the conclusion of this article will be made available by the authors, without undue reservation.

\section{AUTHOR CONTRIBUTIONS}

C-GD and NZ conceived the idea and supervised the work. Y-FZ carried out theory calculations. All authors analyzed the results and contributed to writing the manuscript.

\section{FUNDING}

This paper was supported by the National Key Research and Development Program of China (2017YFA0303403), Shanghai Science and Technology Innovation Action Plan (No. 19JC1416700), Natural Science Foundation of Shanghai (Grant No. 20ZR1418300) and the National Natural Science Foundation of China (No. 11774092, 61790583, 61874043, and 12074119). Computations were performed at the East China Normal University Multifunctional Platform for Innovation.

\section{SUPPLEMENTARY MATERIAL}

The Supplementary Material for this article can be found online at: https://www.frontiersin.org/articles/10.3389/fmats.2021.736057/ full\#supplementary-material

Chang, K., Miller, B. J., Yang, H., Lin, H., Parkin, S. S. P., Barraza-Lopez, S., et al. (2019). Standing Waves Induced by Valley-Mismatched Domains in Ferroelectric SnTe Monolayers. Phys. Rev. Lett. 122 (20), 206402. doi:10.1103/PhysRevLett.122.206402

Deng, Z., Li, Z., Wang, W., and She, J. (2019). Vibrational Properties and Raman Spectra of Pristine and Fluorinated Blue Phosphorene. Phys. Chem. Chem. Phys. 21 (3), 1059-1066. doi:10.1039/c8cp05699d

Ding, J., Shao, D.-F., Li, M., Wen, L.-W., and Tsymbal, E. Y. (2021). TwoDimensional Antiferroelectric Tunnel Junction. Phys. Rev. Lett. 126 (5), 057601. doi:10.1103/PhysRevLett.126.057601

Ding, W., Zhu, J., Wang, Z., Gao, Y., Xiao, D., Gu, Y., et al. (2017). Prediction of intrinsic two-dimensional ferroelectrics in In2Se3 and other III2-VI3 van der Waals materials. Nat. Commun. 8, 14956. doi:10.1038/ncomms14956

Duan, C.-G., Sabirianov, R. F., Mei, W.-N., Jaswal, S. S., and Tsymbal, E. Y. (2006). Interface Effect on Ferroelectricity at the Nanoscale. Nano Lett. 6 (3), 483-487. doi:10.1021/nl052452l 
Fei, R., Kang, W., and Yang, L. (2016). Ferroelectricity and Phase Transitions in Monolayer Group-IV Monochalcogenides. Phys. Rev. Lett. 117 (9), 097601. doi:10.1103/PhysRevLett.117.097601

Feng, Y., Zhou, J., Du, Y., Miao, F., Duan, C.-G., Wang, B., et al. (2015). Raman Spectra of Few-Layer Phosphorene Studied from First-Principles Calculations. J. Phys. Condens. Matter 27 (18), 185302. doi:10.1088/09538984/27/18/185302

Ferrari, A. C., Meyer, J. C., Scardaci, V., Casiraghi, C., Lazzeri, M., Mauri, F., et al. (2006). Raman Spectrum of Graphene and Graphene Layers. Phys. Rev. Lett. 97 (18), 187401. doi:10.1103/PhysRevLett.97.187401

Franzman, M. A., Schlenker, C. W., Thompson, M. E., and Brutchey, R. L. (2010). Solution-Phase Synthesis of SnSe Nanocrystals for Use in Solar Cells. J. Am. Chem. Soc. 132 (12), 4060-4061. doi:10.1021/ja100249m

Garcia, V., Fusil, S., Bouzehouane, K., Enouz-Vedrenne, S., Mathur, N. D., Barthélémy, A., et al. (2009). Giant Tunnel Electroresistance for Nondestructive Readout of Ferroelectric States. Nature 460 (7251), 81-84. doi:10.1038/nature08128

Giannozzi, P., Baroni, S., Bonini, N., Calandra, M., Car, R., Cavazzoni, C., et al. (2009). QUANTUM ESPRESSO: a Modular and Open-Source Software Project for Quantum Simulations of Materials. J. Phys. Condens. Matter 21 (39), 395502. doi:10.1088/0953-8984/21/39/395502

Gonze, X., Stumpf, R., and Scheffler, M. (1991). Analysis of Separable Potentials. Phys. Rev. B 44 (16), 8503-8513. doi:10.1103/PhysRevB.44.8503

Grimme, S. (2006). Semiempirical GGA-type Density Functional Constructed with a Long-Range Dispersion Correction. J. Comput. Chem. 27 (15), 1787-1799. doi:10.1002/jcc.20495

Guan, Z., Hu, H., Shen, X., Xiang, P., Zhong, N., Chu, J., et al. (2020). Recent Progress in Two-Dimensional Ferroelectric Materials. Adv. Electron. Mater. 6 (1), 1900818. doi:10.1002/aelm.201900818

Higashitarumizu, N., Kawamoto, H., Lee, C.-J., Lin, B.-H., Chu, F.-H., Yonemori, I., et al. (2020). Purely In-Plane Ferroelectricity in Monolayer SnS at Room Temperature. Nat. Commun. 11 (1), 2428. doi:10.1038/s41467-020-16291-9

Hohenberg, P., and Kohn, W. (1964). Inhomogeneous Electron Gas. Phys. Rev. 136 (3B), B864-B871. doi:10.1103/PhysRev.136.B864

Huang, C., Du, Y., Wu, H., Xiang, H., Deng, K., and Kan, E. (2018a). Prediction of Intrinsic Ferromagnetic Ferroelectricity in a Transition-Metal Halide Monolayer. Phys. Rev. Lett. 120 (14), 147601. doi:10.1103/PhysRevLett.120.147601

Huang, W., Fang, Y.-W., Yin, Y., Tian, B., Zhao, W., Hou, C., et al. (2018b). SolidState Synapse Based on Magnetoelectrically Coupled Memristor. ACS Appl. Mater. Inter. 10 (6), 5649-5656. doi:10.1021/acsami.7b18206

Junquera, J., and Ghosez, P. (2003). Critical Thickness for Ferroelectricity in Perovskite Ultrathin Films. Nature 422 (6931), 506-509. doi:10.1038/ nature 01501

Kohn, W., and Sham, L. J. (1965). Self-Consistent Equations Including Exchange and Correlation Effects. Phys. Rev. 140 (4A), A1133-A1138. doi:10.1103/ PhysRev.140.A1133

Kong, X., Berlijn, T., and Liang, L. (2021). Thickness and Spin Dependence of Raman Modes in Magnetic Layered Fe 3 GeTe 2. Adv. Electron. Mater. 7, 2001159. doi:10.1002/aelm.202001159

Lee, C., Yan, H., Brus, L. E., Heinz, T. F., Hone, J., and Ryu, S. (2010). Anomalous Lattice Vibrations of Single- and Few-Layer MoS2. ACS Nano 4 (5), 2695-2700. doi: $10.1021 / \mathrm{nn} 1003937$

Lin, L.-F., Zhang, Y., Moreo, A., Dagotto, E., and Dong, S. (2019). Frustrated Dipole Order Induces Noncollinear Proper Ferrielectricity in Two Dimensions. Phys. Rev. Lett. 123 (6), 067601. doi:10.1103/PhysRevLett.123.067601

Liu, F., You, L., Seyler, K. L., Li, X., Yu, P., Lin, J., et al. (2016). Room-temperature Ferroelectricity in CuInP2S6 Ultrathin Flakes. Nat. Commun. 7, 12357. doi:10.1038/ncomms12357

Liu, S.-C., Dai, C.-M., Min, Y., Hou, Y., Proppe, A. H., Zhou, Y., et al. (2021). An Antibonding Valence Band Maximum Enables Defect-Tolerant and Stable GeSe Photovoltaics. Nat. Commun. 12 (1), 670. doi:10.1038/s41467-02120955-5

Mathews, S., Ramesh, R., Venkatesan, T., and Benedetto, J. (1997). Ferroelectric Field Effect Transistor Based on Epitaxial Perovskite Heterostructures. Science 276 (5310), 238-240. doi:10.1126/science.276.5310.238

Morales-Ferreiro, J. O., Diaz-Droguett, D. E., Celentano, D., and Luo, T. (2017). First-Principles Calculations of Thermoelectric Properties of IV-VI
Chalcogenides 2D Materials. Front. Mech. Eng. 3 (15), 15. doi:10.3389/ fmech.2017.00015

Park, M., Choi, J. S., Yang, L., and Lee, H. (2019). Raman Spectra Shift of Few-Layer IV-VI 2D Materials. Sci. Rep. 9, 19826. doi:10.1038/s41598-019-55577-x

Perdew, J. P., Burke, K., and Ernzerhof, M. (1996). Generalized Gradient Approximation Made Simple. Phys. Rev. Lett. 77 (18), 3865-3868. doi:10.1103/PhysRevLett.77.3865

Perdew, J. P., and Zunger, A. (1981). Self-interaction Correction to DensityFunctional Approximations for many-electron Systems. Phys. Rev. B 23 (10), 5048-5079. doi:10.1103/PhysRevB.23.5048

Rodin, A. S., Gomes, L. C., Carvalho, A., and Castro Neto, A. H. (2016). Valley Physics in Tin (II) Sulfide. Phys. Rev. B 93 (4), 045431. doi:10.1103/ PhysRevB.93.045431

Saboori, S., Deng, Z., Li, Z., Wang, W., and She, J. (2019). $\beta$-As Monolayer: Vibrational Properties and Raman Spectra. ACS Omega 4 (6), 10171-10175. doi:10.1021/acsomega.9b00712

Scott, J. F., and Paz de Araujo, C. A. (1989). Ferroelectric Memories. Science 246 (4936), 1400-1405. doi:10.1126/science.246.4936.1400

Shen, X.-W., Fang, Y.-W., Tian, B.-B., and Duan, C.-G. (2019). Two-Dimensional Ferroelectric Tunnel Junction: The Case of Monolayer In:SnSe/SnSe/Sb:SnSe Homostructure. ACS Appl. Electron. Mater. 1 (7), 1133-1140. doi:10.1021/ acsaelm.9b00146

Shen, X.-W., Tong, W.-Y., Gong, S.-J., and Duan, C.-G. (2018). Electrically Tunable Polarizer Based on 2D Orthorhombic Ferrovalley Materials. 2d Mater. 5 (1), 011001. doi:10.1088/2053-1583/aa8d3b

Shi, G., and Kioupakis, E. (2015). Anisotropic Spin Transport and Strong VisibleLight Absorbance in Few-Layer SnSe and GeSe. Nano Lett. 15 (10), 6926-6931. doi:10.1021/acs.nanolett.5b02861

Song, S., Zhang, Y., Guan, J., and Dong, S. (2021). Noncollinear Ferrielectricity and Morphotropic Phase Boundary in Monolayer GeS. Phys. Rev. B 103 (14), L140104. doi:10.1103/PhysRevB.103.L140104

Tan, P. H., Han, W. P., Zhao, W. J., Wu, Z. H., Chang, K., Wang, H., et al. (2012). The Shear Mode of Multilayer Graphene. Nat. Mater. 11 (4), 294-300. doi:10.1038/Nmat3245

Tian, B., Liu, L., Yan, M., Wang, J., Zhao, Q., Zhong, N., et al. (2019). A Robust Artificial Synapse Based on Organic Ferroelectric Polymer. Adv. Electron. Mater. 5 (1), 1800600. doi:10.1002/aelm.201800600

Umari, P., Pasquarello, A., and Dal Corso, A. (2001). Raman Scattering Intensities in $\alpha$-quartz: A First-Principles Investigation. Phys. Rev. B 63 (9), 094305. doi:10.1103/PhysRevB.63.094305

Velev, J. P., Duan, C.-G., Belashchenko, K. D., Jaswal, S. S., and Tsymbal, E. Y. (2007). Effect of Ferroelectricity on Electron Transport inPt/BaTiO3/ PtTunnel Junctions. Phys. Rev. Lett. 98 (13), 137201. doi:10.1103/ PhysRevLett.98.137201

Wang, H., and Qian, X. (2017). Two-dimensional Multiferroics in Monolayer Group IV Monochalcogenides. $2 d$ Mater. 4 (1), 015042. doi:10.1088/2053$1583 / 4 / 1 / 015042$

Wang, X., Li, Y., Huang, L., Jiang, X.-W., Jiang, L., Dong, H., et al. (2017). ShortWave Near-Infrared Linear Dichroism of Two-Dimensional Germanium Selenide. J. Am. Chem. Soc. 139 (42), 14976-14982. doi:10.1021/ jacs.7b06314

Wang, Y., Niranjan, M. K., Janicka, K., Velev, J. P., Zhuravlev, M. Y., Jaswal, S. S., et al. (2010). Ferroelectric Dead Layer Driven by a Polar Interface. Phys. Rev. B 82 (9), 094114. doi:10.1103/PhysRevB.82.094114

Wu, M., and Jena, P. (2018). The rise of two-dimensional van der Waals ferroelectrics. Wires Comput. Mol. Sci. 8 (5), e1365. doi:10.1002/wcms.1365

Xu, B., Deng, J., Ding, X., Sun, J., and Liu, J. Z. (2021). Interlayer Sliding Induced Intralayer Ferroelectric-Antiferroelectric Transition in Bilayer Group-IV Monochalcogenides. arXiv preprint arXiv:2103.12518.

Yagmurcukardes, M., Peeters, F. M., and Sahin, H. (2018). Electronic and Vibrational Properties of PbI2 : From Bulk to Monolayer. Phys. Rev. B 98 (8), 085431. doi:10.1103/PhysRevB.98.085431

Yagmurcukardes, M., Senger, R. T., Peeters, F. M., and Sahin, H. (2016). Mechanical Properties of Monolayer GaS and GaSe Crystals. Phys. Rev. B 94 (24), 245407. doi:10.1103/PhysRevB.94.245407

Yang, S., Liu, Y., Wu, M., Zhao, L.-D., Lin, Z., Cheng, H.-c., et al. (2018). Highlyanisotropic Optical and Electrical Properties in Layered SnSe. Nano Res. 11 (1), 554-564. doi:10.1007/s12274-017-1712-2 
You, L., Zhang, Y., Zhou, S., Chaturvedi, A., Morris, S. A., Liu, F., et al. (2019). Origin of giant negative piezoelectricity in a layered van der Waals ferroelectric. Sci. Adv. 5 (4), eaav3780. doi:10.1126/sciadv.aav3780

Yuan, S., Luo, X., Chan, H. L., Xiao, C., Dai, Y., Xie, M., et al. (2019). Roomtemperature Ferroelectricity in MoTe2 Down to the Atomic Monolayer Limit. Nat. Commun. 10, 1775. doi:10.1038/s41467-019-09669-x

Zhang, X., Qiao, X.-F., Shi, W., Wu, J.-B., Jiang, D.-S., and Tan, P.-H. (2015). Phonon and Raman Scattering of Two-Dimensional Transition Metal Dichalcogenides from Monolayer, Multilayer to Bulk Material. Chem. Soc. Rev. 44 (9), 2757-2785. doi:10.1039/c4cs00282b

Zhao, H., Mao, Y., Mao, X., Shi, X., Xu, C., Wang, C., et al. (2018). Band Structure and Photoelectric Characterization of GeSe Monolayers. Adv. Funct. Mater. 28 (6), 1704855. doi:10.1002/adfm.201704855

Zhou, Y., Wu, D., Zhu, Y., Cho, Y., He, Q., Yang, X., et al. (2017). Out-ofPlane Piezoelectricity and Ferroelectricity in Layered $\alpha$-In2Se3 Nanoflakes. Nano Lett. 17 (9), 5508-5513. doi:10.1021/ acs.nanolett.7b02198
Conflict of Interest: The authors declare that the research was conducted in the absence of any commercial or financial relationships that could be construed as a potential conflict of interest.

Publisher's Note: All claims expressed in this article are solely those of the authors and do not necessarily represent those of their affiliated organizations, or those of the publisher, the editors and the reviewers. Any product that may be evaluated in this article, or claim that may be made by its manufacturer, is not guaranteed or endorsed by the publisher.

Copyright $\odot 2021$ Zhao, Guan, Zhong, Yue, Xiang and Duan. This is an open-access article distributed under the terms of the Creative Commons Attribution License (CC $B Y$ ). The use, distribution or reproduction in other forums is permitted, provided the original author(s) and the copyright owner(s) are credited and that the original publication in this journal is cited, in accordance with accepted academic practice. No use, distribution or reproduction is permitted which does not comply with these terms. 